\title{
Intermittent low dose irradiation enhances the effectiveness of radio- and chemo-therapy for human colorectal adenocarcinoma cell line HT-29
}

\author{
YANG WANG $^{1 *}$, YEZHOU LI $^{2 *}$, LI YANG $^{1}$ and DEXIN YIN ${ }^{2}$ \\ ${ }^{1}$ Department of Gastroenterology, FAW General Hospital, Changchun, Jilin 130011; ${ }^{2}$ Department of Vascular Surgery, \\ China-Japan Union Hospital of Jilin University, Changchun, Jilin 130033, P.R. China
}

Received December 9, 2016; Accepted May 15, 2017

DOI: $10.3892 / o r .2017 .5679$

\begin{abstract}
Low dose irradiation (LDIR) induces hormesis and adaptive response in organism and mammalian cell lines. Notably, LDIR generates distinct biological effects in cancer cells from normal cells, e.g., it may affect the growth of cancer cells via the activation of certain cell signaling pathway, which does not exist in normal cells. Therefore, LDIR is considered as a promising assistant method of clinical cancer therapy. In this study, we chose human colorectal adenocarcinoma cell line HT-29 as the experimental model, and investigated the differential biological effects between $250 \mathrm{mGy}$ single dose LDIR and $250 \mathrm{mGy}$ intermittent LDIR pretreatments in high dose irradiation (HDIR) radiotherapy and 5-fluorouracil (5-FU) based chemotherapy. Through the cell growth assays, we observed that $250 \mathrm{mGy}$ intermittent LDIR pretreatment significantly increased the killing effect of both radiotherapy and chemotherapy. Western blotting results showed that intermittent LDIR pretreatment apparently activated the ATM/p53 (ataxia telangiectasia mutated, ATM) pathway in radiotherapy; it also activated ERK and p38MAPK pathways in chemotherapy. When we used chemical inhibitors to block the ATM/p53 or p38MAPK pathways, the intermittent LDIR induced cell growth inhibitions were reversed. However, blockage of ERK pathway could not affect the cell growth inhibiton in chemotherapy. Taken together, our findings evaluated the intermittent LDIR as a potential valuable method that can enhance the effectiveness of radiotherapy and chemotherapy, especially in the radio- or chemo-resistant tumor types.
\end{abstract}

Correspondence to: Dr Dexin Yin, Department of Vascular Surgery, China-Japan Union Hospital of Jilin University, 126 Xiantai Street, Changchun, Jilin 130033, P.R. China

E-mail: jlucjuhyd@163.com

${ }^{*}$ Contributed equally

Key words: low dose irradiation, colorectal adenocarcinoma, chemotherapy, radiotherapy, cell signaling

\section{Introduction}

Colorectal cancer (CRC) is the third most common cancer in China and has become one of the leading causes of cancerrelated mortality in the developed countries (1-3). Every year, there are approximately 1.2 million new cases diagnosed as CRC worldwide (4).

Chemotherapy and radiotherapy are two important methods for the treatment of CRC. However, radio- and chemo-resistance leading to tumor recurrence and the corresponding poor prognosis, is still a serious concern. Therefore, enhancing radio- and chemo-sensitivity, overcoming radio- and chemo-resistance, and improving the efficiency of radio- and chemo-therapy have great practical significance in the clinical treatment of CRC.

Low dose ionizing radiation (LDIR) is proved having hormesis $(5,6)$ and adoptive effect $(7)$. LDIR generates distinct biological effects in cancer cells from normal cells, e.g., it may affect the growth of cancer cells via the activation of certain cell signaling pathway, which does not exist in normal cells $(8,9)$. However, in which manner LDIR affects the proliferation of cancer cells and the scope of application of LDIR in clinical carcinoma therapy, are still in controversial and needed to be deeply clarified.

In this study, we developed a differential LDIR model. Instead of providing a single dose of $250 \mathrm{mGy}$, we divided the LDIR radiation dose into a ten-time $25 \mathrm{mGy}$ with 3-day intervals in between. Then the intermittent LDIR was used jointly with 2 Gy HDIR radiotherapy or 5-fluorouracil (5-FU) based chemotherapy; we analyzed the cell growth of human colorectal adenocarcinoma cell line HT-29 in these combination therapies and evaluated the therapeutic effectivness of intermittent LDIR. We also investigated the activation of cell signaling pathways after the combination therapies. We hope this observation provides new insight into LDIR and experimental evidence for the multimodal treatment of colorectal cancer.

\section{Materials and methods}

Cell culture and treatments. Human colorectal adenocarcinoma cell line HT-29 was purchased from American Type 
Culture Collection (ATCC, VA, USA). HT-29 was maintained in MyCoy'5A media (Thermo Fisher Scientific, Beijing, China) supplemented with $10 \%$ fetal bovine serum (FBS, Hyclone, Beijing, China) and $1 \%$ antibiotics (penicillin-streptomycin, Invitrogen, CA, USA). Cells were cultured at $37^{\circ} \mathrm{C}$ in a humidified incubator with a constant air flow of $5 \% \mathrm{CO}_{2}$.

ATM inhibitor KU55933 (Selleck, Shanghai, China), p53 inhibitor pifithrin- $\alpha$ (PFT- $\alpha$, Beyotime Institute of Biotechnology, Jiangsu, China), ERK inhibitor PD98059 (Selleck) and p38MAPK inhibitor SB203580 (Selleck) were used to block the function of ATM, p53 and MEK. The final concentration for all the chemical inhibitors is $10 \mu \mathrm{M}$ in the cell culture media.

LDIR strategy. HT-29 cells were ionizing irradiated at the dose rate of $12.5 \mathrm{mGy} / \mathrm{min}$ (for LDIR) or $500 \mathrm{mGy} / \mathrm{min}$ (for HDIR) by X-RAD 320 (Precision X-RAD, North Branford, CT, USA). In order to investigate the intermittent LDIR effect on colorectal cancer, HT-29 cells were divided into three experimental groups. Group A, cells were irradiated for 10 times with a dose of $25 \mathrm{mGy}$ at each time. The time interval between two irradiations was 3 days. The total dose was $250 \mathrm{mGy}$ and the time span was 30 days; group B, cells were parallelly cultured for 30 days and accepted a dose of 250 mGy LDIR on day 30; group C, mock irradiation group as control. After that, cell culture media were replaced and cells were harvested immediately or continually cultured until the next step of the experiment was carried out.

Treatment with chemotherapeutic drug or HDIR. Chemotherapeutic drug 5-FU (Sigma, Shanghai, China) was dissolved in PBS. For drug treatment, appropriate amount of HT-29 colorectal cancer cells from experimental group A, B or C were treated by $50 \mu \mathrm{g} / \mathrm{ml} 5$-FU solution for $24 \mathrm{~h}$. Then, cell culture media were changed and cells were either harvested or used for the proliferation assays. For radiotherapy, appropriate amount of HT-29 cells from group A, B or C were irradiated by 2 Gy HDIR ( $50 \mathrm{mGy} / \mathrm{min})$, and cells were either harvested or used for the proliferation assays.

Cell proliferation assay. WST-1 assay was performed to evaluate cell proliferation. After the treatment by either anticancer drug or HDIR, cell proliferation assays were determined using WST-1 cell proliferation reagent (Beyotime Biotechnology). According to the manufacturer's instructions, $10 \mu \mathrm{l}$ WST- 1 was added to $100 \mu \mathrm{l}$ cell culture medium and incubated at $37^{\circ} \mathrm{C}$ in the dark for $2.5 \mathrm{~h}$. The absorbance of 450 and $630 \mathrm{~nm}$ were measured by microplate reader (Thermo Fisher Scientific). Final OD (optical density) was designated as $\mathrm{OD}_{450}-\mathrm{OD}_{630}-\mathrm{OD}_{\text {blank }}$.

Flow cytometry for cell cycle distribution assay. Approximately $2 \times 10^{6}$ cells were collected and washed by $1 \mathrm{ml}$ cold PBS. Cells were centrifuged and resuspended in $1 \mathrm{ml}$ fixation solution (700 $\mu \mathrm{l}$ ethanol and $300 \mu \mathrm{PBS}$ ). After incubated at $4^{\circ} \mathrm{C}$ for $4 \mathrm{~h}$, cells were washed twice with $1 \mathrm{ml}$ PBS. Cells were pelleted and suspended in $0.5 \mathrm{ml}$ propidium iodide (PI, Sigma) staining solution $(50 \mu \mathrm{g} / \mathrm{ml}$ PI, $20 \mu \mathrm{g} / \mathrm{ml}$ RNase A and $0.2 \%$ Triton $\mathrm{X}-100)$ and incubated in the dark at $37^{\circ} \mathrm{C}$ for $30 \mathrm{~min}$. Cell cycle distribution was analyzed by a BD FACSCalibur flow cytometer (FACScan, BD Biosciences, CA, USA).
Flow cytometry for cell apoptosis. Annexin V-FITC and PI double staining flow cytometry analyses were employed for the assay of cell apoptosis after radiotherapy and chemotherapy. Approximately $1 \times 10^{6}$ HT- 29 cells were plated in a 6-well plate containing $2 \mathrm{ml}$ medium and accepted the treatment of radio- or chemo-therapy. Fourty-eight hours after the treatments, cells were collected in centrifuge tubes, washed three times with cold PBS and binding buffer, and then stained with Annexin V-FITC and PI (Annexin V-FITC Apoptosis Detection kit, BD Bioscience) for apoptosis detection. Briefly, HT-29 cells were first resuspended in binding buffer. Then, $5 \mu 1$ of Annexin V-FITC was added to the tubes and were incubated for $10 \mathrm{~min}$ followed by the addition of $5 \mu 1$ PI (Sigma). After 15-min incubation in PI buffer, cells were immediately analysed using a flow cytometer (BD Biosciences) with the FlowJo FACS analysis software. The cells in the different portions represent the different cell states as follows: the late-apoptotic cells are present in the upper right portion, the viable cells are present in the lower left portion, and the early apoptotic are the cells present in the lower right portion.

Reverse transcription-quantitative polymerase chain reaction $(R T-q P C R)$. Cell total RNA was extracted using RNAzol (Sigma) and the residual genomic DNA was removed by DNase I (Roche, Shanghai, China). M-MLV Reverse Transcriptase (Invitrogen) was used to synthesize cDNA. The mRNA expression level of caspase-3 and p21 was quantified by normalizing over $\beta$-actin. The sequences of the primers used for RT-qPCR were as follows: p21-fwd, 5'-ATGTCCGT CAGAACCCATGC-3'; p21-rev, 5'-AGTCGAAGTTCCATC GCTCAC-3'; caspase-3-fwd, 5'-GATGATGACATGGCGTG TCATA-3'; caspase-3-rev, 5'-AGCGACTGGATGAACCA GGA-3'; $\beta$-actin-fwd, 5'-ATCACCATTGGCAATGAGCG-3'; $\beta$-actin-rev, 5'-CGTCACACTTCATGATGGAGT-3'.

Western blot analysis. Cell total protein was extracted by protein extraction kit (Thermo Fisher Scientific) and the protein concentration was determined by BCA protein assay kit (Bio-Rad). Appropriate quantity of protein was separated by SDS polyacrylamide gel electrophoresis (SDS-PAGE) and then electrophoretically transferred to polyvinylidene difluoride (PVDF) membranes (Millipore, Billerica, MA, USA). The blots were blocked with 5\% non-fat milk in TBST at $37^{\circ} \mathrm{C}$ for $1 \mathrm{~h}$. Then, blots were probed with monoclonal antibodies against ATM (1:500, Abcam, Shanghai, China), p53 (1:1,500, DO7, Santa Cruz Biotechnology, Santa Cruz, CA, USA), p21 (1:500, C-19, Santa Cruz Biotechnology), cyclin A (1:500, Santa Cruz Biotechnology), CDK2 (1:500, Santa Cruz Biotechnology), caspase-3 (1:1,000, Cell Signaling Technology, Beijing, China), cleaved caspase-3 (1:1,000, Cell Signaling Technology), ERK/phos-ERK (1:1,000, Cell Signaling Technology), p38/phos-p38 (1:1,000, Cell Signaling Technology) and $\beta$-actin (1:3,000, Santa Cruz Biotechnology) at $4^{\circ} \mathrm{C}$ overnight. After washed for $3 \times 5 \mathrm{~min}$ in TBST, the blots were incubated with HRP conjugated goat anti-mouse or goat anti-rabbit second antibodies (Santa Cruz) at room temperature for $1 \mathrm{~h}$. After washed for another $3 \times 5 \mathrm{~min}$ in TBST, the blots were visualized by the enhanced chemiluminescence system (ECL; Thermo Fisher Scientific). Protein expression 
A

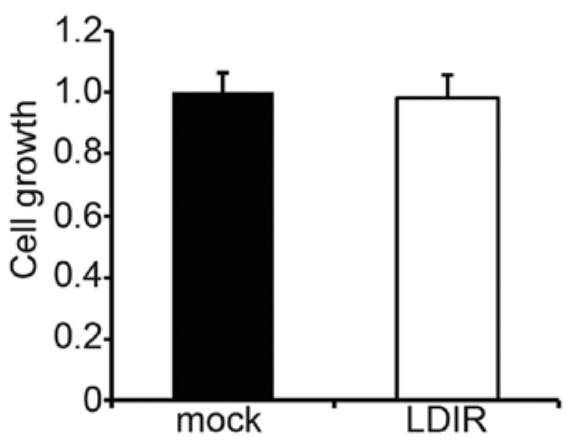

B

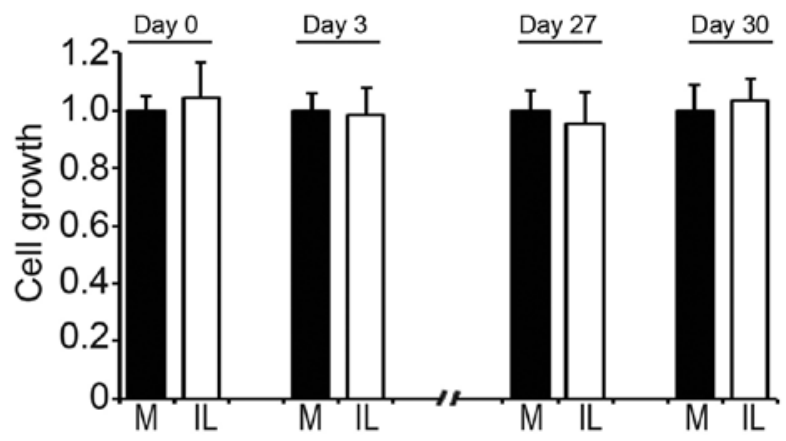

Figure 1. Cell growth status after LDIR or intermittent LDIR. (A) HT-29 cells were administered a single dose of $250 \mathrm{mGy}$ LDIR on day 30 . After that, cell growth was determined by WST-1 assay. (B) HT-29 cells accepted a ten-time intermittent LDIR with 25 mGy each time. After each time of irradiation, cell growth was determined by WST-1 assay. The time interval between two irradiations was 3 days. M, mock irradiation; IL, intermittent LDIR.

was determined semiquantitatively by densitometric analysis with the Quantity One software (Bio-Rad).

Statistical analysis. All experiments were repeated for at least three times. Data and statistics are presented as means \pm SD. The significance was determined by Student's t-test using SPSS 17.0. $\mathrm{P}<0.05$ was considered to indicate a statistically significant difference $\left({ }^{*} \mathrm{P}<0.05 ;{ }^{* *} \mathrm{P}<0.01\right)$.

\section{Results}

Single dose LDIR or intermittent LDIR does not affect the cell growth of HT-29 colorectal cancer cells. To illustrate the cell growth status after LDIR, we used $250 \mathrm{mGy}$ single dose LDIR or $25 \mathrm{mGy}$ ten-times LDIR to irradiate HT-29 colorectal cancer cells. The cell growth was determined by WST-1 kit. We found that neither single dose LDIR (Fig. 1A) nor intermittent LDIR (Fig. 1B, only the results of days 0, 3, 27 and 30 are shown) changed the cell growth of HT-29 (P>0.05).

Intermittent LDIR promotes the therapeutic effects of radiotherapy and chemotherapy. In order to investigate whether the LDIR affects the therapeutic effect of radiotherapy or chemotherapy, HT-29 colorectal cancer cells were pretreated by either single dose LDIR or intermittent LDIR before HDIR or 5-FU chemotherapy. The results of cell growth assay are shown in Fig. 2A. Compared to the non-LDIR or single dose LDIR pretreatments, the intermittent LDIR pretreatment obviously inhibited the cell growth in radiotherapy (Fig. 2A) and chemotherapy (Fig. 2B). The cell morphology showed a similar result (Fig. 2C). We analyzed the cell cycle distrubution by flow cytometry and found that the pretreatment of intermittent LDIR induced a remarkable $\mathrm{S}$ phase arrest in both radiotherapy ( 36.2 vs $27.3 \%$, compared with the single dose LDIR group; Fig. 2D) and chemotherapy (40.4 vs $27.9 \%$, compared with the single dose LDIR group; Fig. 2E). We also used the PI-Annexin V kit to assay cell apoptosis. As can be seen in Fig. 2F, the pretreatment of intermittent LDIR increased cell apoptosis to $46 \%$ in radiotherapy and $49 \%$ in chemotherapy. The apoptosis percentages were markedly higher than in the intermittent LDIR groups (Fig. $2 \mathrm{G}$, $\mathrm{P}<0.01)$.
Intermittent LDIR activates cell cycle arrest and apoptosis pathway. To delineate the mechanism underlying this enhanced cell growth inhibition after intermittent LDIR, we used RT-PCR and western blotting to examine the cell cycle arrest and apoptosis pathway genes for their expression in the HDIR and 5-FU treated cells. We observed that although both the HDIR and 5-FU treatments upregulated the expression of p21 and caspase-3 genes, single dose LDIR pretreatment did not enhance the killing effect of radiotherapy or chemotherapy ( $\mathrm{P}>0.05$, Fig. 3A and $\mathrm{B}$ ). Interestingly, the intermittent LDIR pretreatment markedly increased the expression of $\mathrm{p} 21$ and caspase- 3 compared to single dose LDIR ( $\mathrm{P}<0.01$, Fig. 3A and $\mathrm{B})$. The activation of the cell cycle arrest and apoptosis pathways were also confirmed via the detection of p21, caspase-3 (cleaved caspase-3), CDK2 and cyclin A by western blotting (Fig. 3C-F).

Intermittent LDIR activates ATM/p53 pathway in HDIR radiotherapy. We investigated the radiotherapy most related ATM/p53 pathway in HDIR treated HT-29 cells. Using western blotting, we showed that compared to single dose LDIR, intermittent LDIR pretreatment could activate the ATM/p53 pathway more efficiently and the expression of ATM and p53 proteins were upregulated significantely (Fig. 4A and B). To confirm that the ATM/p53 pathway was specifically activated by intermittent LDIR, ATM and p53 inhibitors were added to the cell culture media prior to the HDIR radiotherapy. Cell growth assay showed that after the ATM/p53 activities were blocked, the intermittent LDIR-induced cell growth inhibition in radiotherapy was abolished $(\mathrm{P}<0.01$, Fig. $4 \mathrm{C})$.

Intermittent LDIR activates ERK and p38MAPK pathways in 5-FU chemotherapy. We also investigated the activity of ERK and p38MAPK pathways in 5-FU chemotherapy. Using western blotting, we demonstrated that compared to 5-FU or single dose LDIR plus 5-FU, the pretreatment of intermittent LDIR increased the expression of ERK/p-ERK (Fig. 5A and B) and p38/p-p38 significantly (Fig. 5C and D). When the p38MAPK pathway was blocked by SB203580, intermittent LDIR-induced cell proliferation inhibition in 5-FU chemotherapy was reversed (Fig. 5E). However, ERK pathway inhibitor PD98059 could not reverse the proliferation inhibition induced by intermittent LDIR in chemotherapy (Fig. 5F). 
A

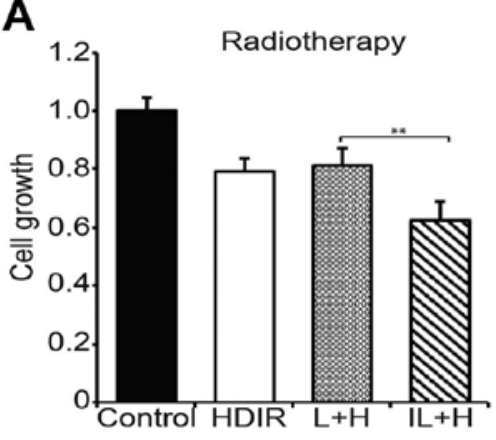

C
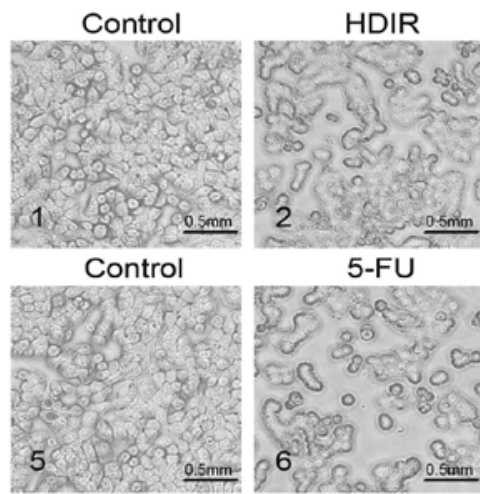

D

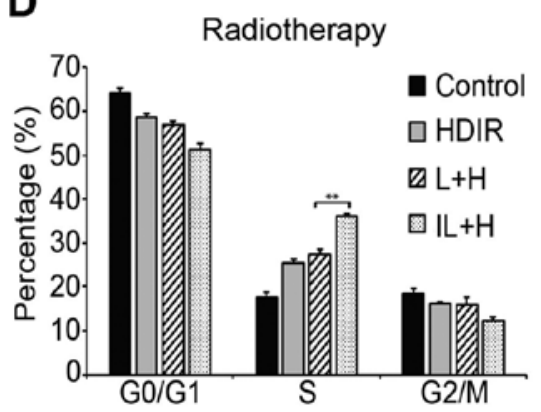

Control

B

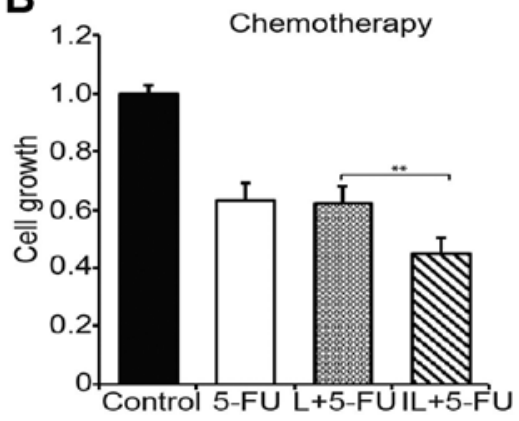

$\mathrm{L}+\mathrm{H}$

$\mathrm{IL}+\mathrm{H}$
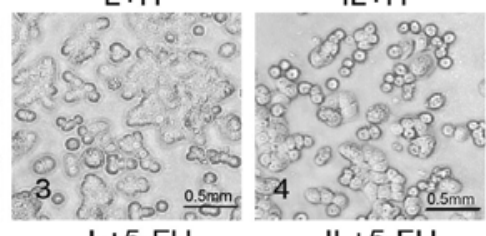

$\mathrm{L}+5-\mathrm{FU}$

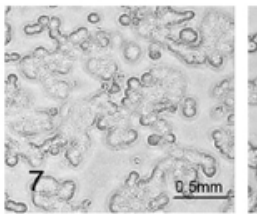

$\mathrm{IL}+5-\mathrm{FU}$

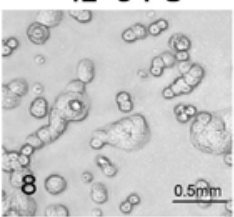

E

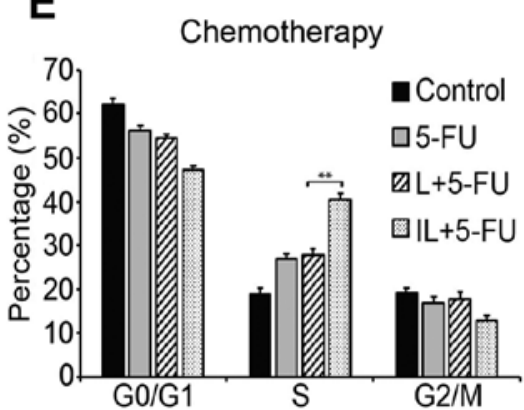

F
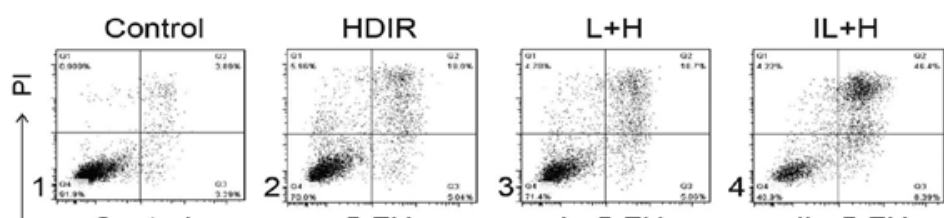

L+5-FU

$\mathrm{IL}+5-\mathrm{FU}$
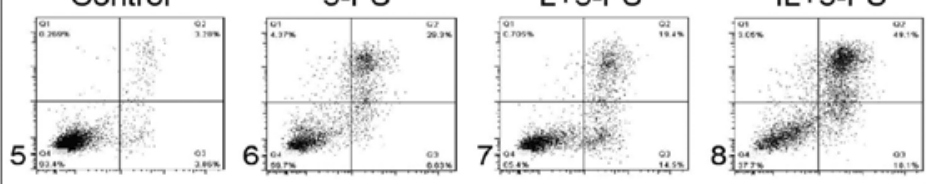

$\longrightarrow$ Annexin-V

$\mathbf{G}$

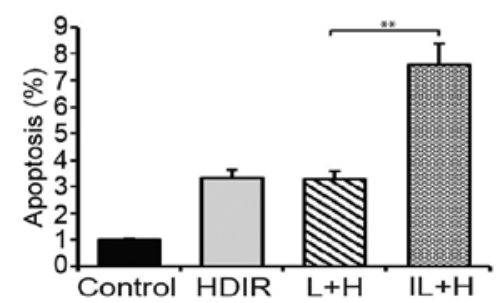

H

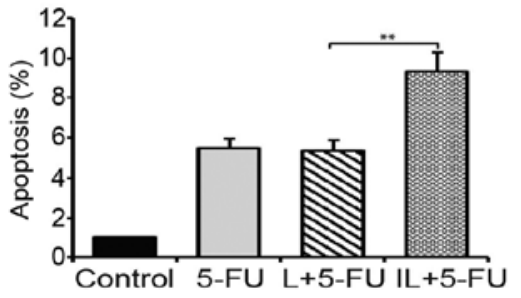

Figure 2. Intermittent LDIR promotes the therapeutic effects of radiotherapy and chemotherapy. (A) HT-29 cells were administered either HDIR, LDIR plus HDIR, or intermittent LDIR plus HDIR. Cell growth was determined by WST-1 assays. (B) HT-29 cells were treated by either 5-FU, LDIR plus 5-FU, or intermittent LDIR plus 5-FU. Cell growth was determined by WST-1 assays. (C) Cell morphology. Plot C1-C4, radiotherapy group. Plot C5-C8, chemotherapy group. (D) Analysis of HT-29 cell cycle distribution by flow cytometry after radiotherapy. (E) Analysis of HT-29 cell cycle distribution by flow cytometry after chemotherapy. (F) Analysis of HT-29 cell apoptosis by flow cytometry. Plot D1-D4, radiotherapy group; Plot D5-D8, chemotherapy group. (G) Cell apoptosis percentage in radiotherapy. (H) Cell apoptosis percentage in chemotherapy. H, HDIR; L, LDIR; IL, intermittent LDIR. ** P $<0.01$ as compared with the single dose LDIR group. 
A

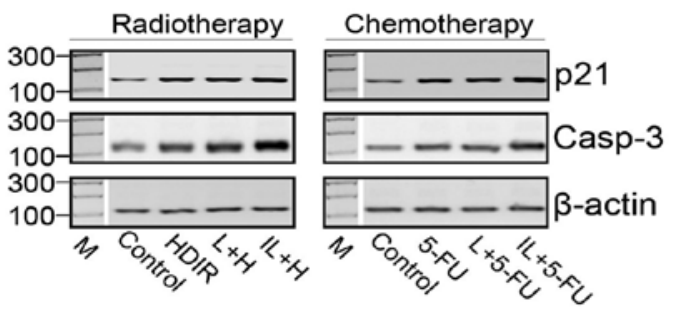

C

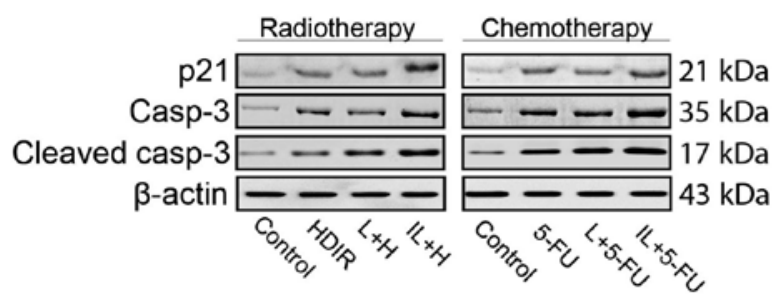

E

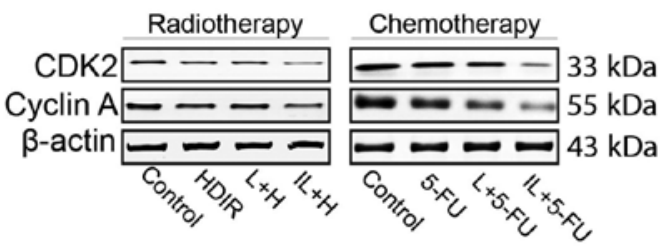

B
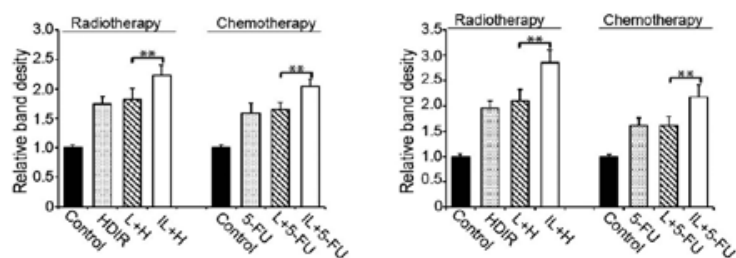

D

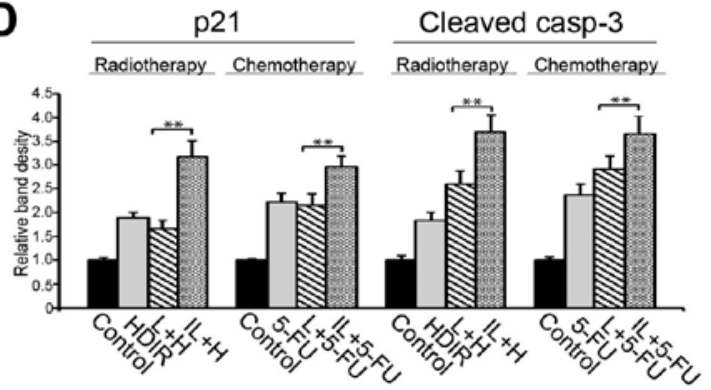

F

CDK2

Cyclin A

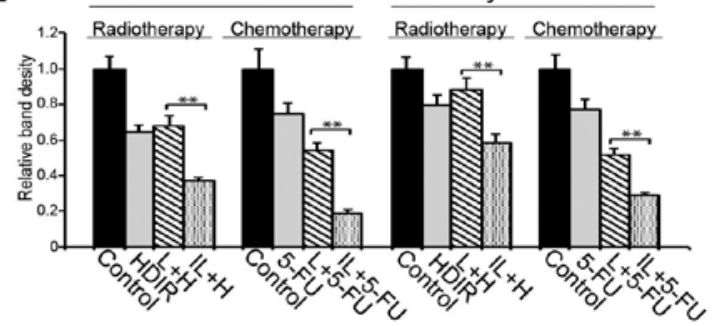

Figure 3. Intermittent LDIR activates cell cycle arrest and the apoptosis pathway. (A) RT-PCR of the cell cycle arrest and apoptosis pathway genes in HT-29 cells. $\beta$-actin was used as the internal control for PCR reaction. (B) Relative band density of RT-PCR. (C) Western blotting of the cell cycle arrest and apoptosis pathway genes in HT-29 cells. $\beta$-actin was used as the internal control for blotting reaction. (D) Relative band density of western blotting. (E) Western blotting of caspase-3 and cyclin-dependent kinase. (F) Relative band density of western blotting. M, 100 bp DNA marker; H, HDIR; L, LDIR; IL, intermittent LDIR. ${ }^{* *} \mathrm{P}<0.01$ as compared with the single dose LDIR group.

A
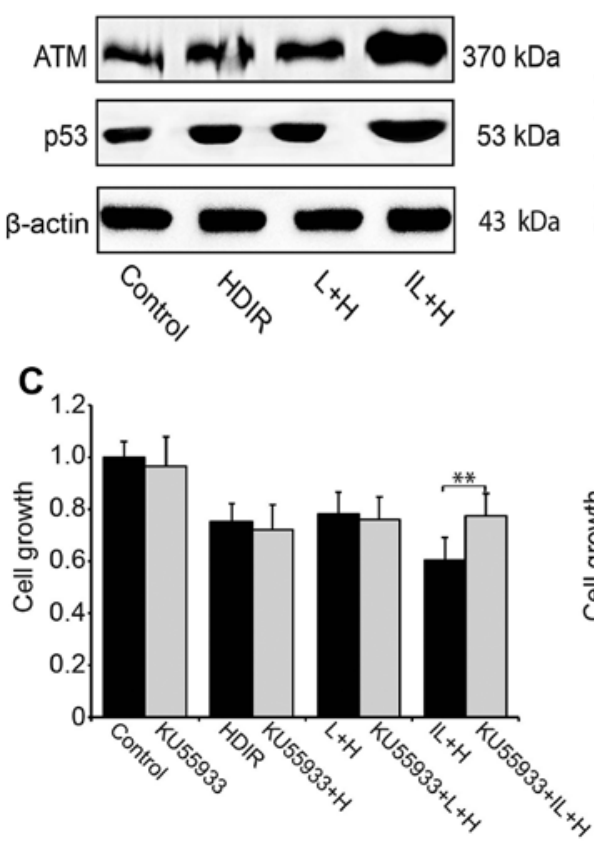

B
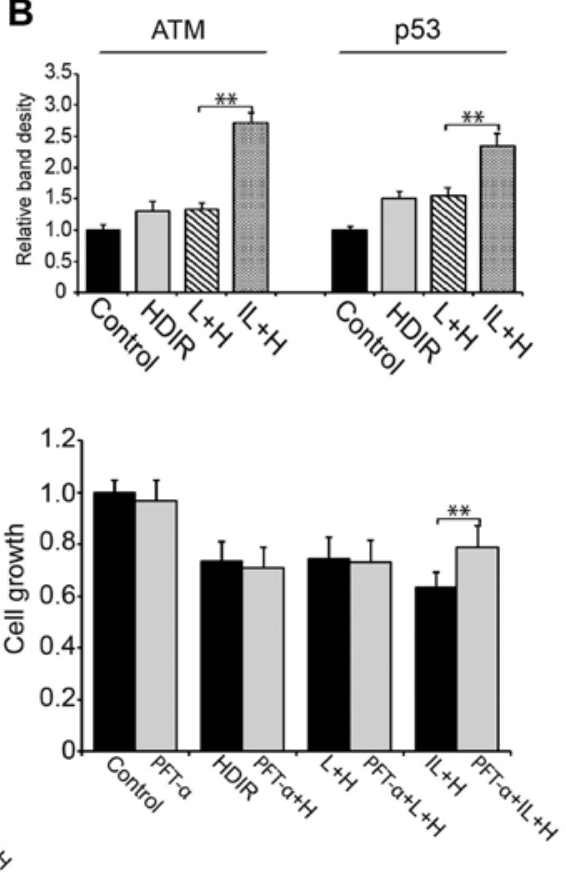

Figure 4. Intermittent LDIR activates ATM/p53 pathway in HDIR radiotherapy. (A) Western blotting of ATM/p53 pathway genes in HT-29 cells. $\beta$-actin was used as the internal control for blotting reaction. (B) Relative band density of western blotting. (C) ATM/p53 pathway inhibitors neutralized the intermittent LDIR induced cell proliferation inhibition in HDIR radiotherapy. In order to inhibit the function of ATM and p53, KU55933 (left panel) and pifithrin- $\alpha$ (right panel) were added to the cell culture media at $24 \mathrm{~h}$ and $2 \mathrm{~h}$ prior to the HDIR radiotherapy. H, HDIR; L, LDIR; IL, intermittent LDIR. ** $\mathrm{P}<0.01$ as compared with the single dose LDIR group. 
A

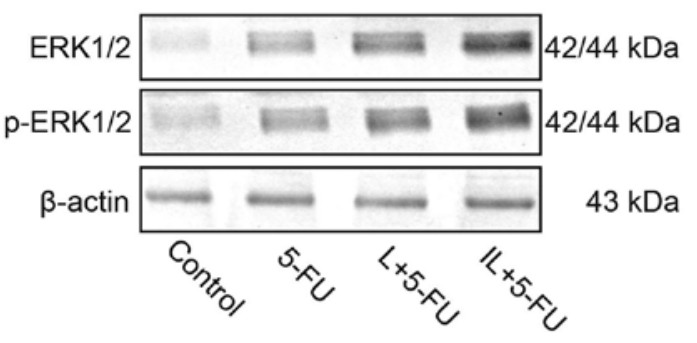

C

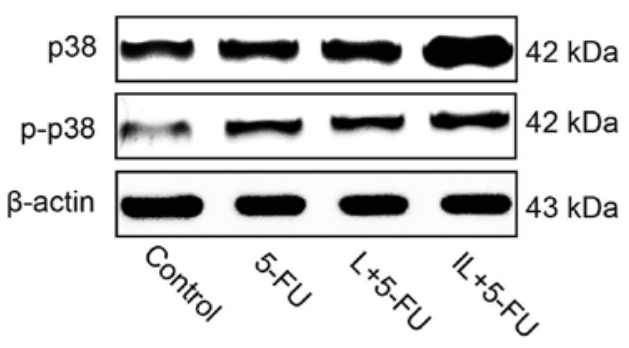

E

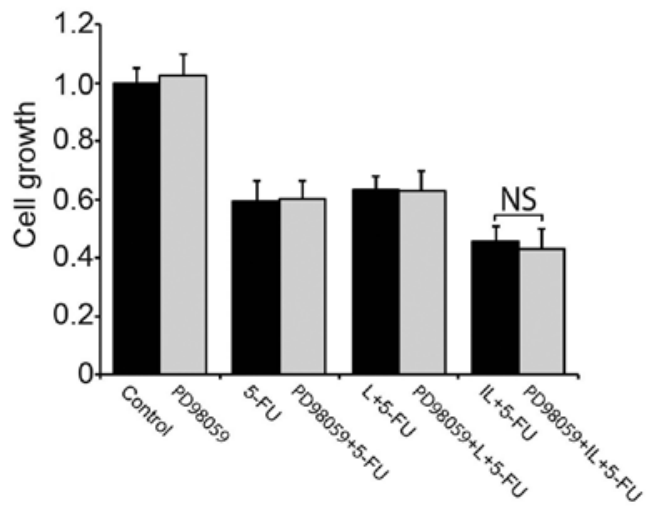

B
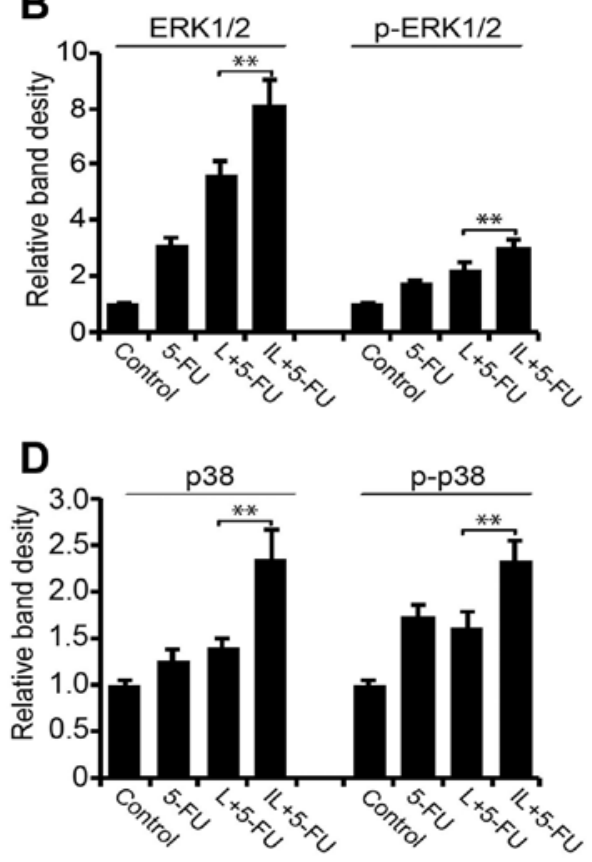

$\mathbf{F}$

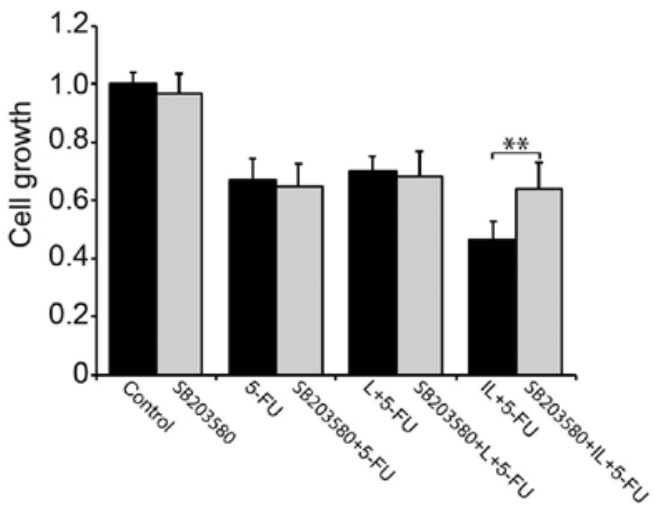

Figure 5. Intermittent LDIR activates ERK and p38MAPK pathways in 5-FU chemotherapy. In order to inhibit the function of ERK and p38MAPK, $10 \mu \mathrm{M}$ PD98059 and SB203580 were added to the cell culture media at $2 \mathrm{~h}$ prior to 5-FU chemotherapy. (A) Western blotting of the MEK/ERK pathway genes in HT-29 cells. $\beta$-actin was used as the internal control for blotting reaction. (B) Relative band density of western blotting. (C) Western blotting of the p38MAPK pathway genes in HT-29 cells. $\beta$-actin was used as the internal control for blotting reaction. (D) Relative band density of western blotting. (E) ERK pathway inhibitors could not reverse the intermittent LDIR induced cell proliferation inhibition. (F) p38MAPK pathway inhibitors neutralized the intermittent LDIR induced cell proliferation inhibition. L, LDIR; IL, intermittent LDIR; NS, not significant. ${ }^{* *} \mathrm{P}<0.01$ as compared with the single dose LDIR group.

\section{Discussion}

Although surgery and chemotherapy are two preferred options in clinical treatment of CRC patients, multimodal treatment approaches are advocated for the therapy of CRC patients in advanced stage. 5 -FU is still the mainstay therapy for patients with advanced CRC, but only $<25 \%$ of patients with advanced CRC show major responses after 5-FU-based chemotherapy. Consequently, resistance to this drug is a major obstacle in CRC chemotherapy (10). Radiotherapy is commonly used to treat multi-tumors to attenuate the risk of recurrence. Growing evidence from clinical trial has proved that an addition of radiotherapy to exclusively surgical treatment of rectal cancer can improve the patient prognosis (11). However, despite impressive initial clinical responses, a large proportion of patients experience resistance to radiotherapy.

In this study, in order to overcome radio- and chemoresistance in the treatment of CRC, a novel combination use of intermittent LDIR with HDIR radiotherapy or 5-FU based chemotherapy was tentatively investigated, and the effectiveness of the pretreatment of LDIR was evaluated. Interestingly, our findings suggest that intermittent LDIR could significantly enhance the sensitivity of the following radiotherapy and chemotherapy. Cell growth assays showed that the pretreatment of intermittent LDIR obviously increased the killing ability of HDIR as well as anticancer drug via a strong induction of cell apoptosis. Then we investigated the involved mechanism on molecular level. Both western blotting and RT-PCR results 
revealed that intermittent LDIR pretreatment stimulated the expression of cell cycle arrest and cell apoptotic genes, and it activated ATM/p53 pathway in radiotherapy and p38MAPK pathway in chemotherapy more efficiently than single dose LDIR. When we treated HT-29 cells with chemical inhibitors of ATM/p53 and p38MAPK pathway to block the cell signaling, the cell growth inhibition induced by the combination therapy strategy was abolished.

5-FU is widely used as the first-line systemic chemotherapy drug. It is an analog of uracil and exerts its anticancer effects through blocking the normal synthesis of DNA and disrupting RNA processing, and eventually lead to apoptosis in the cancer cells $(12,13)$. So far, various causes have been found to contribute to 5-FU resistance, such as activation of the JNK pathway (14), overexpression of Bcl-2 and Bcl-X (15), therapy-induced autophagy (16), and MAPK pathway (17). de la Cruz-Morcillo et al demonstrated that inhibition of p38MAPK correlates with a decrease in the 5-FU-associated apoptosis and chemical resistance in colorectal cancer cells (17). Our study is in agreement with de la Cruz-Morcillo et al; both the two studies proved that it is the p38MAPK rather than the other signaling pathways, e.g., ERK pathway, that controls the cellular response to 5-FU.

ATM and p53 are two of the most important genes that are involed in cellular response to radiotherapy and chemotherapy. Yang et al found that the activation of ATM was the initiating event in LDR induced hormesis and adaptive response, and the distinct activation of ATM/AKT/GSK-3 $\beta$ signaling pathway may explain the differential biological effects between lung cancer cells and normal lung epithelial cells (18). Brazina et al showed that the interplay between ATM, p53 and DAXX plays a key role in the regulation of ionizing radiation or genotoxic drug-induced DNA damage (19). Lin et al found that in chronic lymphocytic leukemia, ATM/p53/p21 pathway defects are strongly associated with chemoresistance or early relapse (20). In our study, a marked upregulation of ATM and p53 was observed after the combined use of intermittent LDIR and HDIR radiotherapy, and we confirmed that the activation of ATM/p53 pathway is a crucial event in the regulation of cell apoptosis during the radiotherapy of HT-29 cells.

In conclusion, this study demonstrates that a combination use of intermittent LDIR and HDIR or 5-FU is a valuable method in promoting the therapeutic effectiveness of radiotherapy and chemotherapy, and this effect is dependent on the activation of ATM/p53 and p38MAPK pathways on molecular level, which controls the cell cycle progression and cell apoptosis. Of importance, since tumor cells are more likely to be genetically heterogeneous, different cells with different genetic or epigenetic backgrounds may respond distinctly to the same kind of radiation even at the same dose level.

\section{References}

1. Su XL, Wang YF, Li SJ, Zhang F and Cui HW: High methylation of the SEPT9 gene in Chinese colorectal cancer patients. Genet Mol Res 13: 2513-2520, 2014.
2. Yang XD, Xu XH, Zhang SY, Wu Y, Xing CG, Ru G, Xu HT and Cao JP: Role of miR-100 in the radioresistance of colorectal cancer cells. Am J Cancer Res 5: 545-559, 2015.

3. Tang FR and Loke WK: Molecular mechanisms of low dose ionizing radiation-induced hormesis, adaptive responses, radioresistance, bystander effects, and genomic instability. Int J Radiat Biol 91: 13-27, 2015.

4. Guo M and Dou J: Advances and perspectives of colorectal cancer stem cell vaccine. Biomed Pharmacother 76: 107-120, 2015.

5. Luckey TD: Physiological benefits from low levels of ionizing radiation. Health Phys 43: 771-789, 1982.

6. Feinendegen LE: Evidence for beneficial low level radiation effects and radiation hormesis. Br J Radiol 78: 3-7, 2005.

7. Olivieri G, Bodycote J and Wolff S: Adaptive response of human lymphocytes to low concentrations of radioactive thymidine. Science 223: 594-597, 1984.

8. Yang G, Li W, Jiang H, Liang X, Zhao Y, Yu D, Zhou L, Wang G, Tian H, Han F, et al: Low-dose radiation may be a novel approach to enhance the effectiveness of cancer therapeutics. Int J Cancer 139: 2157-2168, 2016.

9. Liang X, Gu J, Yu D, Wang G, Zhou L, Zhang X, Zhao Y, Chen X, Zheng S, Liu Q, et al: Low-dose radiation induces cell proliferation in human embryonic lung fibroblasts but not in lung cancer cells: Importance of ERK1/2 and AKT signaling pathways. Dose Response 14: 1559325815622174, 2016.

10. Shin YK, Yoo BC, Hong YS, Chang HJ, Jung KH, Jeong SY and Park JG: Upregulation of glycolytic enzymes in proteins secreted from human colon cancer cells with 5-fluorouracil resistance. Electrophoresis 30: 2182-2192, 2009.

11. Hafner MF and Debus J: Radiotherapy for colorectal cancer: Current standards and future perspectives. Visc Med 32: 172-177, 2016.

12. Longley DB, Harkin DP and Johnston PG: 5-fluorouracil: Mechanisms of action and clinical strategies. Nat Rev Cancer 3: 330-338, 2003.

13. Grem JL: 5-Fluorouracil: Forty-plus and still ticking. A review of its preclinical and clinical development. Invest New Drugs 18: 299-313, 2000.

14. Sui X, Kong N, Wang X, Fang Y, Hu X, Xu Y, Chen W, Wang K, Li D, Jin W, et al: JNK confers 5-fluorouracil resistance in p53-deficient and mutant p53-expressing colon cancer cells by inducing survival autophagy. Sci Rep 4: 4694, 2014.

15. Violette S, Poulain L, Dussaulx E, Pepin D, Faussat AM, Chambaz J, Lacorte JM, Staedel C and Lesuffleur T: Resistance of colon cancer cells to long-term 5-fluorouracil exposure is correlated to the relative level of $\mathrm{Bcl}-2$ and $\mathrm{Bcl}-\mathrm{X}(\mathrm{L})$ in addition to Bax and p53 status. Int J Cancer 98: 498-504, 2002.

16. Wei MF, Chen MW, Chen KC, Lou PJ, Lin SY, Hung SC, Hsiao M, Yao CJ and Shieh MJ: Autophagy promotes resistance to photodynamic therapy-induced apoptosis selectively in colorectal cancer stem-like cells. Autophagy 10: 1179-1192, 2014.

17. de la Cruz-Morcillo MA, Valero ML, Callejas-Valera JL, AriasGonzález L, Melgar-Rojas P, Galán-Moya EM, García-Gil E, García-Cano J and Sánchez-Prieto R: P38MAPK is a major determinant of the balance between apoptosis and autophagy triggered by 5 -fluorouracil: Implication in resistance. Oncogene 31: 1073-1085, 2012.

18. Yang G, Yu D, Li W, Zhao Y, Wen X, Liang X, Zhang X, Zhou L, $\mathrm{Hu}$ J, Niu C, et al: Distinct biological effects of low-dose radiation on normal and cancerous human lung cells are mediated by ATM signaling. Oncotarget 7: 71856-71872, 2016.

19. Brazina J, Svadlenka J, Macurek L, Andera L, Hodny Z, Bartek J and Hanzlikova H: DNA damage-induced regulatory interplay between DAXX, p53, ATM kinase and Wip1 phosphatase. Cell Cycle 14: 375-387, 2015.

20. Lin K, Adamson J, Johnson GG, Carter A, Oates M, Wade R, Richards S, Gonzalez D, Matutes E, Dearden C, et al: Functional analysis of the ATM-p53-p21 pathway in the LRF CLL4 trial: Blockade at the level of p21 is associated with short response duration. Clin Cancer Res 18: 4191-4200, 2012. 